\title{
Chronic Kidney Disease in Patients Undergoing Cardiac Device Placement: Results of a Retrospective Study
}

\author{
Mohammad A. Hossain ${ }^{\mathrm{a}}$, Firas Ajamª, Hetavi Mahida ${ }^{\mathrm{a}}$, Anas Alrefaee ${ }^{\mathrm{a}}$, Swapnil Patel ${ }^{\mathrm{a}}$, \\ Khushboo Agarwal ${ }^{\mathrm{a}}$, Marjan Alidoost ${ }^{\mathrm{a}}$, Shereen Dahab ${ }^{\mathrm{a}}$, Amy Quinlan ${ }^{\mathrm{a}}$, \\ Michael Orange ${ }^{\mathrm{a}}$, Arman Mushtaq ${ }^{\mathrm{a}}$, Arif Asifa, b
}

\begin{abstract}
Background: Cardiovascular issues (especially arrhythmia and sudden cardiac death) are one of the most common causes of mortality in patients with chronic kidney disease (CKD). To minimize cardiac mortality, these patients frequently require various cardiac devices, such as pacemakers, loop recorders, and defibrillators which can compromise their vascular access. In this study, we aim to determine the prevalence of CKD in patients undergoing cardiac device placement and their progression of CKD.
\end{abstract}

Methods: Institutional review board approval was obtained for this study. A total of 688 patients undergoing cardiac device placement were included in this study over a 3-year period at Jersey Shore University Medical Center. Demographic characteristics, comorbidities, base-line renal functions during the procedure, types of cardiac devices, sites of vascular access and follow-up renal function when available were assessed retrospectively. Patients were categorized into CKD stages 1 - 5 based on the National Kidney Foundation-Kidney Disease Outcomes Quality Initiative (NKF-KDOQI) guidelines. The patients who were already on hemodialysis were excluded in this study.

Results: The average age of the patient were 73.9 years with male predominance $(60 \%)$. A total of 227 patients $(33 \%)$ had estimated glomerular filtration rate $(\mathrm{eGFR})<60 \mathrm{~mL} / \mathrm{min}$ consistent with the evidence of advanced-stage CKD (stages 3 - 5) at the time of cardiac device placement. The most common types of device placements were new insertion/replacement of atrial and ventricular leads $(39.5 \%)$, loop recorder implantation $(21.1 \%)$ and generator changes on an already implanted device (11\%). Only 4\% (28/688) had a leadless cardiac device placement. The most common access sites were subclavian $(47.1 \%)$, axillary $(32.3 \%)$ and femoral $(12.2 \%)$.

Manuscript submitted January 8, 2020, accepted January 23, 2020

aDepartment of Medicine, Jersey Shore University Medical Center, Hackensack-Meridian School of Medicine at Seton Hall, Neptune, NJ 07753, USA

${ }^{\mathrm{b}}$ Corresponding Author: Arif Asif, Department of Medicine, Jersey Shore University Medical Center, Hackensack-Meridian School of Medicine at Seton Hall, 1945 Route 33, Neptune, NJ 07753, USA.

Email: arif.asif@hackensackmeridian.org

doi: https://doi.org/10.14740/jocmr4075
Conclusions: The present study demonstrated that nearly one-third of the patient undergoing cardiac device placement had an advanced degree of renal failure. Because CKD is a progressive disease, many of these patients might require renal replacement therapy in the future. Transvenous devices is not a good choice in this group of patients as they will ultimately require an arteriovenous fistula. Subcutaneous leadless cardiac device insertion might be a better option in patients with advanced CKD.

Keywords: Chronic kidney disease; Cardiac device; Arrhythmia

\section{Introduction}

Chronic kidney disease (CKD) affects 30 million people or $15 \%$ of the US population [1]. Cardiovascular issues (especially arrhythmia and sudden cardiac death) are one of the most common causes of mortality in patients with CKD [2,3]. To mitigate cardiac complications, these patients often require various cardiac devices, such as pacemakers, loop recorders and defibrillators. The placement of these devices poses multiple predicaments in renal failure patients. Many of these implantable cardiac devices have leads that can cause central venous stenosis and thrombosis which is very critical for renal patients as compared to the general population [4-8]. Because CKD is a progressive disease, these patients depend on patent central venous access for the creation of an arteriovenous (AV) fistula in the near future [4]. Loss of this venous access can be an important factor for their ultimate survival. In addition to that, abnormal renal function is an independent risk factor for cardiac implantable electronic device (CIED) infection [9-11]. In this study, we aim to determine the prevalence of CKD in patients undergoing cardiac device placement and their progression of CKD.

\section{Materials and Methods}

A total of 688 patients who underwent cardiac device placement at Jersey Shore University Medical Center over a 3-year period (June 2015 to June 2018) were included in this study. Demographic characteristics, comorbidities, base-line renal functions at the time procedure, types of cardiac device, and 
insertion sites were assessed retrospectively. Estimated glomerular filtration rate (eGFR) was calculated by the Chronic Kidney Disease Epidemiology Collaboration (CKD-EPI) creatinine equation for all of the patients to ascertain the degree of advanced CKD. Advanced CKD was defined as CKD stage greater than 3 or more. Patients were categorized into CKD stages 1 - 5 based on the National Kidney Foundation Dialysis Outcomes Quality Initiative (NKF-KDOQI) guidelines [12].

Institutional review board approval was obtained for this study. All study procedures were carried out in accordance with the Declaration of Helsinki regarding research involving human subjects. The summary statistics of continuous variables were reported as mean \pm standard deviation.

\section{Results}

A total of 688 patients underwent cardiac device placement at Jersey Shore University Medical Center over a 3-year period. Demographic characteristics revealed that the average age of the patient was 73.9 years with male predominance $(60 \%)$ (Table 1). Major comorbidities included hypertension (76.2\%), hyperlipidemia (66.4\%), cardiovascular disease (44.4\%) and congestive heart failure (44.9\%) (Table 1). Most common types of device placements were new insertion or replacement of atrial and ventricular leads (39.5\%), loop recorder implantation $(21.1 \%)$ and generator changes on an already implanted devices (11\%), and only $4 \%(28 / 688)$ had leadless cardiac device placement (Table 2). The most common insertion sites were subclavian (47.1\%), axillary $(32.3 \%)$ and femoral $(12.2 \%)$. A total of 227 patients $(33 \%)$ had evidence of stage 3 - 5 CKD (stage $3 \mathrm{~A}$ GFR $=45$ - $59 \mathrm{~mL} / \mathrm{min}(\mathrm{n}=116)$; stage
Table 1. Demographics of Patient Undergoing Cardiac Device Placement $(n=688)$

\begin{tabular}{|c|c|}
\hline Age & 73.9 years \\
\hline \multicolumn{2}{|l|}{ Gender } \\
\hline Men & $414(60 \%)$ \\
\hline Women & $274(40 \%)$ \\
\hline \multicolumn{2}{|l|}{ Race } \\
\hline White & $636(92.4 \%)$ \\
\hline Black & $40(5.8 \%)$ \\
\hline Asian & $4(0.6 \%)$ \\
\hline Hispanic & $3(0.4 \%)$ \\
\hline Other & $5(0.7 \%)$ \\
\hline Congestive heart failure & $309(45 \%)$ \\
\hline Diabetes & $165(24 \%)$ \\
\hline Renal disease & $227(33 \%)$ \\
\hline Dialysis & $20(3 \%)$ \\
\hline Cardiovascular disease & $399(58 \%)$ \\
\hline Cerebrovascular accident & $114(17 \%)$ \\
\hline Peripheral vascular disease & $46(7 \%)$ \\
\hline Lung disease & $135(20 \%)$ \\
\hline Hyperlipidemia & $457(66 \%)$ \\
\hline Hypertension & $524(76 \%)$ \\
\hline
\end{tabular}

3B GFR $=30-44 \mathrm{~mL} / \mathrm{min}(\mathrm{n}=69)$; stage 4 GFR $=15-29 \mathrm{~mL} /$ $\min (\mathrm{n}=23)$; stage $5 \mathrm{GFR}<15 \mathrm{~mL} / \mathrm{min}(\mathrm{n}=19))$ at the time of

Table 2. Types of Cardiac Device and Sites of Access $(n=688)$

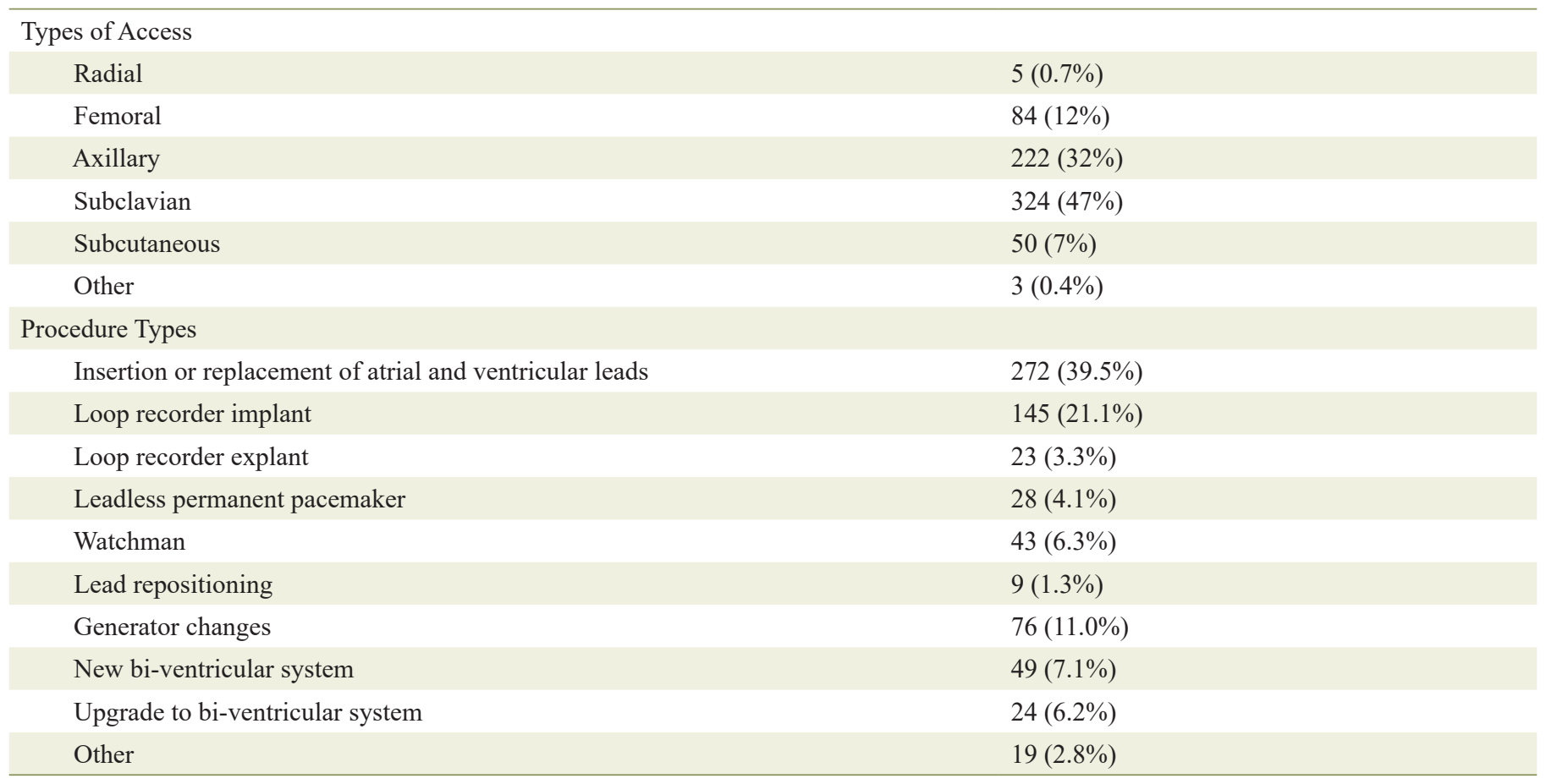


Table 3. Stages of CKD in Patients' Who Underwent Cardiac Device Placement

\begin{tabular}{lll}
\hline Stages of CKD & Number of patients & eGFR $^{\mathbf{a}}$ \\
\hline Stage 3A & $116(17 \%)$ & $45-59 \mathrm{~mL} / \mathrm{min}$ \\
Stage 3B & $69(10 \%)$ & $30-44 \mathrm{~mL} / \mathrm{min}$ \\
Stage 4 & $23(3 \%)$ & $15-29 \mathrm{~mL} / \mathrm{min}$ \\
Stage 5 & $19(3 \%)$ & $<15 \mathrm{~mL} / \mathrm{min}$ \\
\hline
\end{tabular}

Stage 1 and 2 CKD are not included.CKD: chronic kidney disease; eGFR: estimated glomerular filtration rate. ${ }^{a}$ eGFR reported by labs > $60 \mathrm{~mL} / \mathrm{min}$.

cardiac device placement (Table 3).

\section{Discussion}

This study finds that CKD stages 3 - 5 presented in nearly $33 \%$ of patients undergoing cardiac device implantation. Our results are consistent with the previous study that demonstrated a similar prevalence of CKD in patient undergoing CIED removal [13].

Recognizing the high prevalence of advanced CKD in this population is important to minimize the risk of device infection. The most common sites of insertion for the cardiac device were subclavian $(47.1 \%)$, axillary $(32.3 \%)$ and femoral $(12.2 \%)$. Almost $80 \%$ of the patient had their device placed through central veins. While placing cardiac devices through central veins is a common pathway, the leads can cause central vein stenosis (CVS) and carry the risk of tricuspid regurgitation by valve adhesion, perforation or entanglement $[5-8,14]$. This point is critically important as many patients will progress to end-stage renal disease and require an AV fistula [10]. The placement of $\mathrm{AV}$ fistula requires the preservation of the vascular system in an upper extremity. In our study, a high prevalence of CKD patients who underwent a cardiac device procedure is at a higher likelihood of requiring hemodialysis. The development of central venous stenosis may prevent the creation of the life time (i.e. AV fistula) for a dialysis patient.

To preserve the central vascular access in CKD patient who are planning to have cardiac devices, placement of the leadless cardiac device and the use of the epicardial route is an important alternative to avoid the transvenous pathway. The validity of this approach was provided by recent studies [15-18]. Data from epicardial studies emphasized that the effectiveness and survival of epicardial leads are essentially the same as the endocardial leads [15-18]. Recently, a subcutaneous approach has also been reported in hemodialysis patients [16-18]. In our study only 4\% (28/688) patients had leadless implanted cardiac devices (ICDs) implanted. Leadless ICDs do not use central veins and are located in the subcutaneous tissue. In this way, they avoid the risk of CVS.

\section{Conclusions}

Our study showed that a significant amount of patients under- going cardiac device placement had advanced CKD. Because CKD is a progressive disease, many of these patients might require renal replacement therapy in the future. Transvenous devices is not a good choice in this group of patients as they cause CVS. This complication can prevent the creation of an AV fistula. The subcutaneous leadless cardiac device may be a better option in a patient with advanced CKD.

\section{Acknowledgments}

We are grateful to the Department of Cardiology at Jersey Shore University Medical Center for their cooperation in this study.

\section{Financial Disclosure}

This project was not supported by any grant or funding agencies.

\section{Conflict of Interest}

The authors declare that there is no conflict of interest regarding the publication of this paper.

\section{Informed Consent}

Not applicable.

\section{Author Contributions}

Each author has individually been involved in and has made substantial contributions to conception and design, acquisition of data, analysis, interpretation of data, drafting and editing the manuscript. MAH and AA contributed to conceptualization and methodology; MAH, FA, HM, AA, KA, MA, SD, $\mathrm{AQ}$, and $\mathrm{MO}$ were involved in investigation and data curation; SP contributed to formal analysis and validation; $\mathrm{MAH}$ and $\mathrm{AM}$ contributed to writing (original draft preparation); AA contributed to writing (reviewing and editing the manuscript); AA and MAH contributed to supervision and visualization; all authors have given final approval of the version to be published.

\section{Data Availability}

The authors declare that data supporting the findings of this study are available within the article.

\section{References}

1. Zhang B, Sun XJ, Ju CH. Thrombolysis with alteplase 
4.5-6 hours after acute ischemic stroke. Eur Neurol. 2011;65(3):170-174.

2. Green D, Roberts PR. Ventricular arrhythmias and sudden death in patients with chronic kidney disease. J Ren Care. 2010;36(Suppl 1):54-60.

3. Cai Q, Mukku VK, Ahmad M. Coronary artery disease in patients with chronic kidney disease: a clinical update. Curr Cardiol Rev. 2013;9(4):331-339.

4. Santoro D, Benedetto F, Mondello P, Pipito N, Barilla D, Spinelli F, Ricciardi CA, et al. Vascular access for hemodialysis: current perspectives. Int J Nephrol Renovasc Dis. 2014;7:281-294.

5. Kusztal M, Nowak K. Cardiac implantable electronic device and vascular access: Strategies to overcome problems. J Vasc Access. 2018;19(6):521-527.

6. Teruya TH, Abou-Zamzam AM, Jr., Limm W, Wong L, Wong L. Symptomatic subclavian vein stenosis and occlusion in hemodialysis patients with transvenous pacemakers. Ann Vasc Surg. 2003;17(5):526-529.

7. Tompkins C, McLean R, Cheng A, Brinker JA, Marine JE, Nazarian S, Spragg DD, et al. End-stage renal disease predicts complications in pacemaker and ICD implants. J Cardiovasc Electrophysiol. 2011;22(10):1099-1104.

8. Asif A, Salman L, Carrillo RG, Garisto JD, Lopera G, Barakat U, Lenz O, et al. Patency rates for angioplasty in the treatment of pacemaker-induced central venous stenosis in hemodialysis patients: results of a multi-center study. Semin Dial. 2009;22(6):671-676.

9. Bloom H, Heeke B, Leon A, Mera F, Delurgio D, Beshai J, Langberg J. Renal insufficiency and the risk of infection from pacemaker or defibrillator surgery. Pacing Clin Electrophysiol. 2006;29(2):142-145.

10. Asif A, Carrillo R, Garisto JD, Lopera G, Ladino M, Barakat U, Eid N, et al. Epicardial cardiac rhythm devices for dialysis patients: minimizing the risk of infection and preserving central veins. Semin Dial. 2012;25(1):88-94.
11. Lekkerkerker JC, van Nieuwkoop C, Trines SA, van der Bom JG, Bernards A, van de Velde ET, Bootsma M, et al. Risk factors and time delay associated with cardiac device infections: Leiden device registry. Heart. 2009;95(9):715720.

12. Inker LA, Astor BC, Fox CH, Isakova T, Lash JP, Peralta CA, Kurella Tamura M, et al. KDOQI US commentary on the 2012 KDIGO clinical practice guideline for the evaluation and management of CKD. Am J Kidney Dis. 2014;63(5):713-735.

13. Asif A, Carrillo R, Garisto JD, Monrroy M, Khan RA, Castro H, Merrill D, et al. Prevalence of chronic kidney disease in patients undergoing cardiac rhythm device removal. Semin Dial. 2013;26(1):111-113.

14. Ailawadi G, Lapar DJ, Swenson BR, Maxwell CD, Girotti ME, Bergin JD, Kern JA, et al. Surgically placed left ventricular leads provide similar outcomes to percutaneous leads in patients with failed coronary sinus lead placement. Heart Rhythm. 2010;7(5):619-625.

15. Bardy GH, Smith WM, Hood MA, Crozier IG, Melton IC, Jordaens L, Theuns D, et al. An entirely subcutaneous implantable cardioverter-defibrillator. N Engl J Med. 2010;363(1):36-44.

16. Vachharajani TJ, Salman L, Costanzo EJ, Mehandru SK, Patel M, Calderon DM, Mathew RO, et al. Subcutaneous defibrillators for dialysis patients. Hemodial Int. 2018;22(1):4-8.

17. Maradey JA, Jao GT, Vachharajani TJ. Leadless pacemaker placement in a patient with chronic kidney disease: A strategy to preserve central veins. Hemodial Int. 2018;22(4):E57-E59.

18. Dhamija RK, Tan H, Philbin E, Mathew RO, Sidhu MS, Wang J, Saour B, et al. Subcutaneous implantable cardioverter defibrillator for dialysis patients: a strategy to reduce central vein stenoses and infections. Am J Kidney Dis. 2015;66(1):154-158. 